\title{
ESTUDO BIBLIOMÉTRICO SOBRE A RECUSA FAMILIAR DE DOAÇÃO DE ÓRGÃOS E TECIDOS PARA TRANSPLANTES NO PERÍODO DE 1990 A 2004
}

\author{
Bibliometric study on family refusal of tissue and organ donation for transplants from 1990 to 2004
}

\author{
Edvaldo Leal de Moraes, Maria Cristina Komatsu Braga Massarollo
}

\begin{abstract}
RESUMO
Introdução: A escassez de órgãos para transplante vem preocupando a sociedade no mundo todo, sendo que a recusa familiar é apontada como um dos fatores limitantes para suprir a crescente demanda do número de transplantes. Objetivos: Este estudo teve como objetivo identificar a produção científica nacional e internacional sobre a recusa familiar; apresentar a produção científica identificada segundo a quantidade, ano de publicação, país de origem e categorizar a produção científica segundo os temas identificados. Método: Para a realização do estudo bibliométrico, utilizaram-se as bases de dados MEDLINE e LILACS, usando como descritores: doação de órgãos x recusa família ou organ donation x family refusal. Resultados: Foram encontrados 52 artigos na MEDLINE e um artigo na LILACS também indexado na MEDLINE. O estudo mostrou que os Estados Unidos foram o principal país que publicou produção científica sobre o assunto (48,1\%), e foi também o país que mais produziu trabalhos sobre recusa familiar na doação de órgãos (21,1\%). Conclusão: Os resultados obtidos mostram que a produção cientifica internacional sobre a recusa familiar tem aumentado, e os Estados Unidos foi o país que mais publicou trabalhos. Por outro lado, países da América Latina, como o Brasil, têm produzido poucos trabalhos sobre o tema (1,9\%), evidenciando a necessidade de aumentar a produção científica nesse campo.
\end{abstract}

Descritores: Transplante de Órgãos, Doação de Órgãos, Recusa Familiar, Enfermagem.

\section{Instituição:}

Departamento de Orientação Profissional da Escola de Enfermagem da Universidade de São Paulo

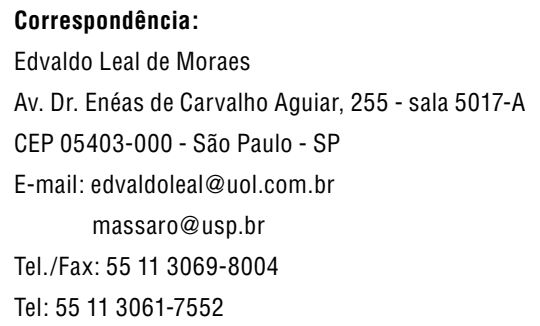

\section{INTRODUÇÃO}

A necessidade de órgãos e tecidos para transplante é muito grande e a demanda supera em muito a oferta. De acordo com os dados da Organização Nacional de Procura de Órgãos (OPTN), nos Estados Unidos da América (EUA) morriam em media 17 pessoas por dia em 2004 à espera de um órgão para transplante, e a cada 12 minutos, uma pessoa entrava na lista de espera. Isso ainda acontece, pois não existem órgãos para todos os que deles necessitam. ${ }^{1}$ Conforme dados da Secretaria de Saúde, em 2006 no estado de São Paulo, a mortalidade em lista de espera para coração foi de $50 \%$, pulmão $48 \%$ e fígado $20 \%{ }^{2}$ Nesse sentido, torna-se cada vez mais urgente melhorar o aproveitamento de potenciais doadores de órgãos e tecidos. Muitos são os obstáculos, sendo que a recusa familiar vem sendo apresentada como uma das causas da diminuição de disponibilização de órgãos de doadores falecidos.

Os motivos mais freqüentes de recusa familiar para doação de órgãos e tecidos para transplante são: (1) desconhecimento do diagnóstico de morte encefálica; (2) desconhecimento do desejo do falecido; (3) inadequação da entrevista familiar na solicitação da doação; (4) problemas com a integridade ou imagem do corpo após a extração dos órgãos e tecidos; (5) questões religiosas; e (6) recusa em vida por parte do falecido. ${ }^{3}$ 
O desconhecimento do diagnóstico de morte encefálica por parte da população tem contribuído muito para a manutenção dos elevados índices de recusa familiar, pois a morte encefálica não é considerada morte, e em $100 \%$ das recusas, $40 \%$ das famílias não aceitam a morte encefálica como morte real. Dessa forma, a recusa da doação pode esconder a negação da morte. ${ }^{4}$

As causas identificadas de recusa, em muitos estudos incluem falta de entendimento pelas famílias do conceito de morte encefálica e suas implicações. ${ }^{5}$

A falta de conhecimento sobre o desejo da pessoa em vida com relação à doação de órgãos e tecidos também representa um problema na doação. Uma pesquisa realizada pela Universidade de Cleveland revelou que o desconhecimento do desejo do falecido contribuiu para a recusa, porém, quando a preferência do doador era conhecida, a probabilidade de doação era 6,9 vezes maior, em comparação com os que desconheciam o desejo da pessoa em vida. ${ }^{6}$ Estudo realizado no Brasil, país que tem como legislação a doação consentida, apresentou resultado semelhante, mostrando que o conhecimento prévio da vontade do falecido foi importante na tomada de decisão familiar. ${ }^{7}$ Existe uma forte associação na decisão familiar para aceitar ou negar a doação, com as seguintes variáveis: conhecimento do desejo do falecido em relação à doação, clima do relacionamento, nível de satisfação da família com o atendimento médico recebido, ${ }^{8}$ além do esclarecimento da definição de morte encefálica e possibilidade de acompanhamento do potencial doador pelos familiares na realização dos procedimentos para confirmação da morte encefálica. ${ }^{9}$

A necessidade de transplante de órgãos aumentou em $200 \%$ na última década, enquanto o número de doadores de órgãos e tecidos tem permanecido relativamente constante. ${ }^{10}$ Isso se deve aos avanços tecnológicos e ao sucesso do transplante nas duas últimas décadas, o que vem resultando num aumento da necessidade de doadores de órgãos. Assim, os centros transplantadores e as Organizações de Procura de Órgãos (OPO) estão enfrentando mundialmente uma escassez de doadores de órgãos. ${ }^{11}$

Isso representa um grande problema, pois o transplante é o tratamento disponível para muitos pacientes com a doença do órgão em estágio final, podendo oferecer a única esperança de sobrevivência. Infelizmente, a concretização dos transplantes é limitada pela escassez de órgãos. Como resultado, muitas pessoas com falência irreversível do órgão morrem esperando por um órgão não disponível, e a recusa familiar representa um dos obstáculos na obtenção de órgãos para transplante. ${ }^{12}$ No Brasil, segundo dados do Registro Brasileiro de Transplantes, em 2006 a não-autorização familiar foi de $34,1 \%$ e a contra-indicação médica ficou em $48,1 \%{ }^{13}$

No transplante de fígado, por exemplo, os candidatos a esse procedimento possuem má qualidade de vida e vivem na perspectiva de morte iminente. Assim, a inclusão do paciente em lista de espera para transplante hepático representa a possibilidade de sobreviver e a possibilidade de melhorar sua qualidade de vida. Entretanto, a espera prolongada por um órgão para transplante propicia o aparecimento de uma série de complicações para o paciente, tornando-o de alto risco para o procedimento e sendo responsável por um número considerável de óbito de pacientes em lista de espera. ${ }^{14}$

Estudo realizado no Reino Unido revelou que o maior obstáculo para a doação de órgãos estava relacionado ao alto índice de recusa familiar. ${ }^{15}$ Pesquisa semelhante realizada nos Estados Unidos mostrou que a recusa familiar foi a principal causa da não efetivação dos doadores potenciais em doadores efetivos. ${ }^{16}$

Assim, pela importância do tema, houve interesse em conhecer a produção científica a respeito, motivando a realização deste trabalho com os seguintes objetivos: Identificar a produção científica nacional e internacional sobre recusa familiar; apresentar a produção científica identificada segundo a quantidade, ano de publicação, país de origem e categorizar a produção científica segundo os temas identificados.

\section{MÉTODO}

A bibliometria é utilizada para quantificar os processos de comunicação escrita e o emprego de indicadores bibliométricos para medir a produção científica. Sua utilização é justificada pelos seguintes motivos: analisar o crescimento, distribuição e tamanho da bibliografia científica e analisar os meios de formação, divulgação e utilização da literatura científica. ${ }^{17}$

Este estudo bibliométrico enfocou a elaboração de uma análise da produção científica referente à recusa familiar na doação de órgãos e tecidos para transplante do período de 1990 a 2004, que posteriormente passou por um processo de distribuição por ano de publicação, país de origem, categorização dos temas e tipo de instituição (universidade, hospital e hospital universitário).

Para o levantamento da produção científica, foram utilizadas as seguintes bases de dados:

- MEDLINE - Base de dados da literatura internacional produzida pela National Library of Medicine - NLM, que reúne referências bibliográficas e resumos de mais de 3700 revistas biomédicas publicadas nos Estados Unidos e em 70 outros países.

- LILACS - Literatura Latino-Americana e do Caribe em Ciências da Saúde, é uma base de dados cooperativa do Sistema BIREME, e compreende a literatura relativa às Ciências da Saúde publicada nos países da região a partir de 1982.

Para levantamento da produção científica foram utilizados os seguintes descritores:

- doação de órgãos x recusa família;

- organ donation x family refusal.

Após obtenção dos resumos, foram realizadas a análise e síntese do material através de: (1) Leitura informativa e exploratória, que consistiu na leitura dos resumos encontrados; (2) Leitura seletiva através da seleção do material quanto à sua pertinência ao estudo e, após a leitura, foi construída uma tabela apresentando o ano e o país da publicação, país de origem, tipo de instituição, título e resumo do trabalho, e (3) Leitura reflexiva, que consistiu na análise dos trabalhos, com a finalidade de compreender e analisar os aspectos abordados pelos autores.

Após a leitura de todo material, os dados foram digitados e apresentados em gráficos, permitindo melhor visualização. 


\section{RESULTADOS}

Na base de dados LILACS, quando utilizados os descritores selecionados para a busca, foi encontrado apenas um artigo indexado referente ao tema em questão, que, por sua vez, também estava indexado na base de dados MEDLINE. Quando utilizada a base de dados MEDLINE, foram encontrados 52 artigos sobre doação de órgãos e recusa familiar.

Assim, para a realização deste estudo foram considerados 52 registros, uma vez que, quando o mesmo trabalho apareceu em mais de uma base, este foi contado apenas uma vez.

Figura 1: Distribuição da produção científica sobre recusa familiar na doação de órgãos e tecidos para transplante, em intervalos de cinco anos, no período de 1990 a 2004.

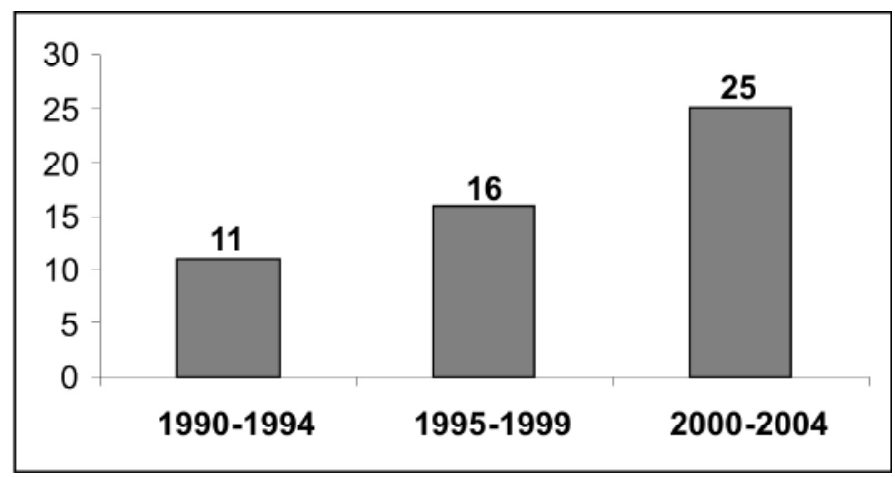

O período submetido à análise bibliométrica está compreendido entre os anos de 1990 a 2004, dividido em intervalos de cinco anos para a apresentação dos dados. Como pode ser observado na Figura 1, nos anos de 1990 a 1994 foram identificados 11 trabalhos $(21,1 \%)$, de 1995 a 1999 foram identificados 16 trabalhos $(30,8 \%)$ e de 2000 a 2004 foram produzidos 25 trabalhos, correspondendo a $48,1 \%$ de toda a produção científica produzida nos últimos 15 anos. Pode-se considerar uma tendência de crescimento na produção científica sobre recusa familiar na doação de órgãos e tecidos no período estudado, indicando uma preocupação cada vez maior com a falta de órgãos de doador falecido para serem disponibilizados aos procedimentos de transplante.
No levantamento realizado, observou-se que a distribuição da produção científica sobre o tema recusa familiar na doação de órgãos e tecidos para transplante em função do país da publicação dos trabalhos revelou os Estados Unidos (EUA) como o principal país que publica produção científica sobre o assunto. Em função dos resultados obtidos, os EUA representaram $48,1 \%$ de toda produção científica, seguido pela França com 15,4\%, Inglaterra 11,5\%, Austrália 5,8\%, Itália e Irlanda com 3,9\% cada.

Os demais países isoladamente, como Espanha, Bélgica, Canadá, Israel, Venezuela e Brasil representaram 1,9\% cada de toda publicação indexada na base de dados Medline.

Em relação ao país de origem, pode-se observar na Figura 2 que os EUA foram o país que mais produziu trabalhos com $21,1 \%$ de toda produção científica, seguido pela França com 15,4\%, Espanha 11,5\%, Inglaterra 7,8\%, Austrália 5,8\%, Itália e Países Baixos 3,9\% cada; os demais países, como o Brasil, representaram apenas $1,9 \%$ da produção cada um e, país de origem não identificado (NI), 11,5\%.

Na categorização dos temas identificados, a Figura 3 mostra que, em $50 \%$ dos trabalhos publicados, os autores apontaram a recusa familiar como sendo um dos fatores responsáveis pela escassez de órgãos e tecidos para transplante. Em 19,2\% dos trabalhos, os autores trataram dos obstáculos que contribuíram para a nãoefetivação da doação; $13,5 \%$ da recusa familiar foram associados a fatores culturais, sócio-econômicos e conhecimento da família sobre o tema. Em 9,6\% os trabalhos não tratavam especificamente

Figura 3: Distribuição por categorias de produção científica sobre a recusa familiar na doação de órgãos e tecidos para transplante no período de 1990 a 2004

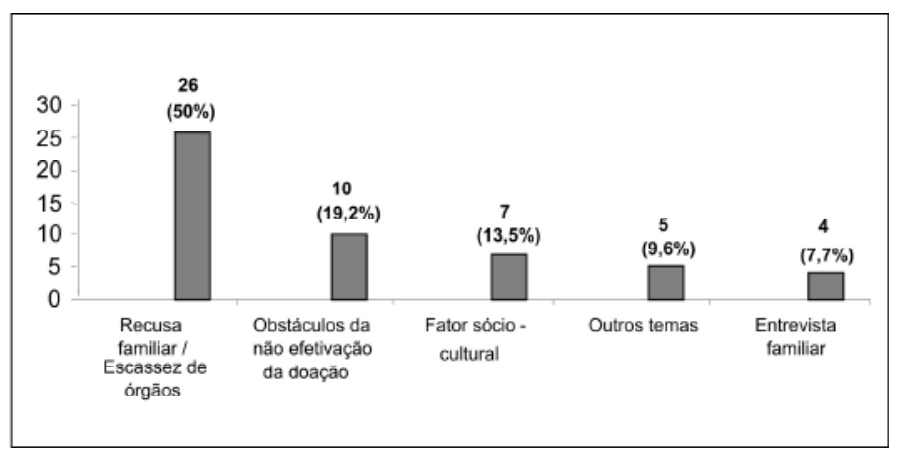

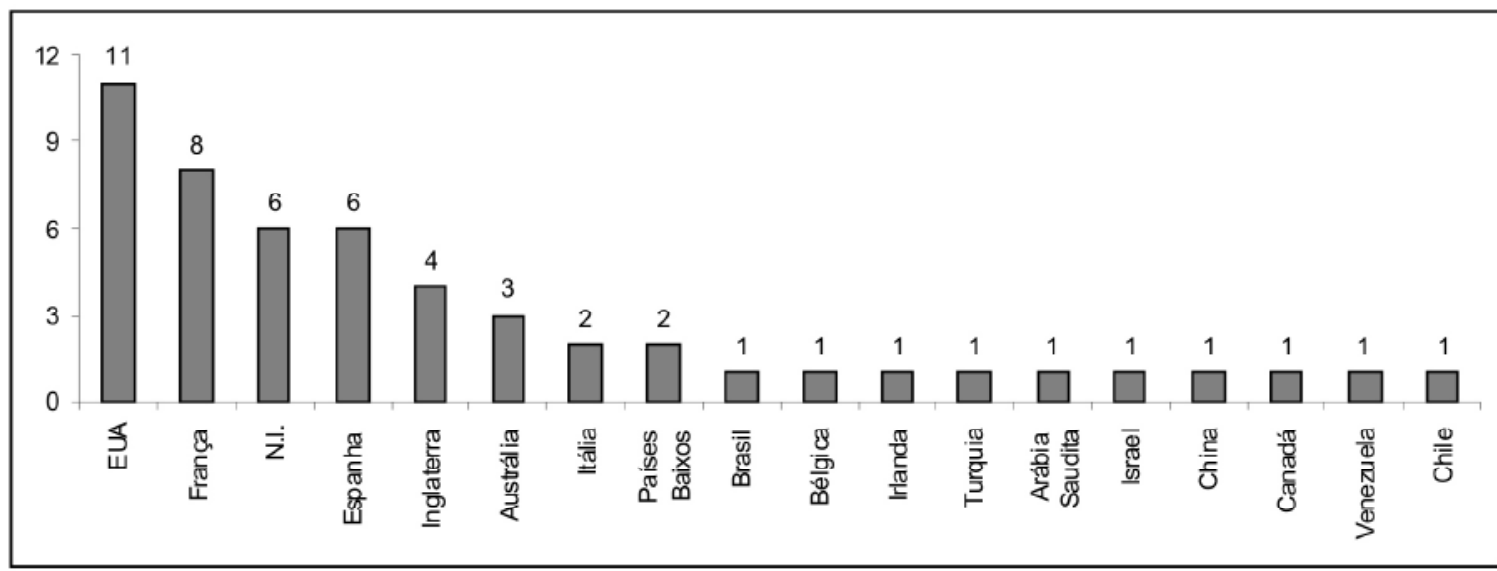

Figura 2: Distribuição segundo a procedência da produção científica sobre a recusa familiar na doação de órgãos e tecidos para transplante no período de 1990 a 2004 
da recusa familiar, mas sim da percepção dos vários profissionais de saúde e da população em relação à doação de órgãos e, em 7,7\% a recusa familiar foi associada a fatores da entrevista familiar.

\section{DISCUSSÃO}

O número insuficiente de doadores para atender à crescente demanda de pacientes em lista de espera representa um dos obstáculos para a realização de transplantes. A grande limitação do transplante com doador falecido é que apenas uma pequena fração dos indivíduos que morrem converte-se em doadores de órgãos e tecidos. Portanto, a falta de órgãos reflete não apenas a falta de potenciais doadores, mas também a falha em convertê-los em doadores efetivos. Sendo assim, o transplante tornou-se vítima de seu próprio sucesso, com um crescente distanciamento entre a demanda para transplante e a disponibilidade de órgãos e tecidos. ${ }^{18}$

É nesse contexto de falta de órgãos e tecidos e na crescente demanda de pacientes em lista de espera que a recusa familiar vem sendo apontada como um dos fatores limitantes na disponibilização de órgãos e tecidos para transplante.

No presente estudo, observou-se um crescimento na produção científica internacional referente à recusa familiar no processo de doação de órgãos e tecidos. Os EUA foi o país que mais publicou $(48,1 \%)$ e produziu $(21,1 \%)$ trabalhos referentes ao tema em questão. Por outro lado, países da América Latina, como o Brasil têm apresentado uma participação muito incipiente $(1,90 \%)$ do total da pesquisa indexada.

$\mathrm{Na}$ categorização do material levantado, a recusa familiar foi apontada como um dos fatores responsáveis pela escassez de órgãos e tecidos para transplante, com $50 \%$ de toda a produção científica. A demanda de órgãos para transplante continua aumentando, embora os esforços das Organizações de Procura de Órgãos (OPO) contra a possível diminuição do número de potenciais doadores, tenham encontrado na recusa familiar uma das causas da desproporção entre o número de potenciais doadores e o número de doadores efetivos. ${ }^{16}$ Os trabalhos apontaram vários motivos para a recusa familiar, sendo que os que mais influenciaram os familiares no momento de decidir sobre a doação foram: desejo do falecido manifestado em vida contrário à doação, desconhecimento do desejo do falecido com relação à doação, falta de compreensão do conceito de morte encefálica, grau de satisfação da família com o atendimento médico prestado ao potencial doador, medo de mutilação do corpo do falecido, negação da morte, falta de consenso familiar e crenças religiosas. Através do levantamento realizado, observa-se uma crescente necessidade por parte dos profissionais envolvidos no processo de doação-transplante de apontar os motivos da recusa familiar. Estudo realizado pela Organização de Procura de Órgãos da Santa Casa de Misericórdia de São Paulo evidenciou que a recusa familiar foi a principal causa da não efetivação da doação. Os motivos que levaram os familiares a recusar a doação foram: negativa expressa em vida, dificuldade em aceitar ou compreender o diagnóstico de morte encefálica, falha no Sistema de Saúde, desconhecimento da opinião do falecido, crenças religiosas e/ou culturais. ${ }^{19}$ Outro trabalho desenvolvido na Grande Recife, PE encontrou motivos de recusa familiar semelhantes aos apresentados nas pesquisas internacionais. ${ }^{20}$ Fica evidente a necessidade de educação dirigida ao público em geral através de campanhas educativas e esclarecimentos pelos meios de comunicação em massa, com o intuito de possibilitar uma mudança de atitude da população em relação à doação de órgãos e tecidos para transplante.

$\mathrm{Na}$ segunda categoria, os estudos revelaram alguns fatores que contribuíram para a não-efetivação dos potenciais doadores, além da recusa familiar. Os obstáculos apontados foram: dificuldade para executar os exames de morte encefálica, falta de interesse do staff da UTI em confirmar o diagnóstico de morte encefálica, a não solicitação da doação, contra-indicação médica, parada cardíaca, e excessiva carga de trabalho na UTI. No Brasil, no ano de 2005 a contra-indicação médica (CIM) representou 47,6\% e a não autorização familiar (NAF) $36,7 \%$ das causas de nãoefetivação da doação. ${ }^{21}$ Outro obstáculo para a não-efetivação da doação está representado pela parada cardíaca. Isso ocorre porque, em muitos casos, os potenciais doadores são notificados muito tardiamente. É preciso melhorar a notificação e a manutenção dos potenciais doadores, pois de cada dez potenciais doadores, somente um é notificado, o que faz com que a média do Brasil seja de apenas seis potenciais doadores por milhão de habitantes por ano. ${ }^{22} \mathrm{~A}$ alta taxa de perda de potenciais doadores de órgãos e tecidos deve-se, em parte, às próprias equipes médicas, que os mantêm sob tratamento médico inadequado e ineficaz para a situação do doente. ${ }^{23}$

Na categorização, fatores sócio-culturais, $13,46 \%$ da produção científica enfatizaram que esses fatores podem influenciar na decisão da doação. Os fatores citados estavam relacionados ao nível educacional e econômico da população, religião e a valores culturais como o apego ao corpo. No entanto, o que se observa é que crenças culturais, mais do que as religiosas opõem-se à doação, pois as religiões são favoráveis à doação. $\mathrm{O}$ apego ao corpo ou à sua integridade e o medo de sua violação é um determinante sócio-cultural que reflete a falta de informação da população. ${ }^{19}$

Em $7,69 \%{ }^{4}$, os trabalhos estudaram fatores relacionados à entrevista familiar que poderiam ser modificados, objetivando diminuir a recusa familiar, tais como: participação do coordenador da OPO na solicitação da doação dos órgãos; a solicitação da doação só deveria ser iniciada quando a família tivesse entendido e aceitado a morte encefálica; proporcionar ambiente privado e calmo na solicitação da doação; buscar entender as necessidades da família no momento de aflição. Na vivência dos profissionais que trabalham diariamente com o processo de doação-transplante, a entrevista é um dos momentos mais estressantes, requerendo preparo e habilidade para lidar com a morte e o sofrimento. Pouco se conhece a respeito do processo de solicitação e sobre o que afeta a vontade da família em doar. Um fator decisivo para a doação é o conhecimento da família sobre o desejo da pessoa com relação à doação. ${ }^{19}$

Dos 52 artigos pesquisados, $9,62 \%{ }^{5}$ trataram de temas referentes à percepção de profissionais de saúde em relação à doação de órgãos; doação em vida e tomada de decisão na doação em vida.

\section{CONCLUSÃO}

Os resultados obtidos revelaram que a produção científica internacional sobre a recusa familiar aumentou nos últimos anos, e os Estados Unidos foram o país que mais publicou trabalhos, com $48,1 \%$ de toda produção. Por outro lado, países da América 
Latina, como o Brasil, têm produzido poucos trabalhos sobre o tema (1,9\%), evidenciando a necessidade de aumentar a produção científica nesse campo.

Fica evidente que o Brasil precisa produzir mais trabalhos indexados referentes ao tema em questão, pois a recusa familiar também representa um obstáculo na obtenção de órgãos e tecidos para transplante, ao lado de outras causas de igual importância, como a baixa taxa de notificação dos potenciais doadores às Centrais de Notificação, Captação e Distribuição de Órgãos (CNCDOs), o alto índice de parada cardíaca que reflete a condição inadequada na manutenção dos potenciais doadores, bem como a contra-indicação médica à doação.

\section{ABSTRACT}

The lack of organs for transplantation have been concerning societies all over the world because the family refusal is a limiting factor to face the increasing demand on the high number of transplantation. Purposes: This study had as target to identify the national and international amount of scientific production on the family refusal, even considering the identified scientific production of quantity, year of publishing, country of origin, and categorizing the scientific production on the identified themes. Methods: To get the bibliometric study performed; the Medline and Lilacs databases having the organ donation X family refusal as describers were used. Results: Fifty-two (52) MEDLINE articles, and one (1) LILACS article also indexed in Medline were found. The study has shown that USA is the main publisher country of the intellectual production on this subject (48.1\%) and the country which mostly produced works on family refusal of organ donation (21.1\%). Conclusion: The results attained show that the international scientific production on family refusal has increased, and the United States is the country with the majority of works published. On the other hand, Latin America countries, such as Brazil, have produced few works on the subject (1.9\%), evidencing the need to increase the scientific production in this field.

Descriptors: Organ Transplantation, Organ Donation, Family Refusal, Nursing.

\section{REFERÊNCIAS}

1. Organ Procurement and Transplantation Network (OPTN). In: http://www.optn.org/ about/transplantation/transplantprocess.asp. (10/abril/2006)

2. São Paulo (Estado). Secretaria de Estado da Saúde. Sistema Estadual de Transplantes. In: Central de Transplantes: http://www.saude.sp.gov.br; 2006.

3. Frutos MA, Blanca MJ, Rando B, Rosel J. Actitudes de las familias de donantes y no-donantes de órganos. Rev Espanhola Transpl. 1994;3:163-9.

4. Fontana R, Almasia A. On accepting death: languages of access to organ sharing. Transplant Proc. 1996;28:392.

5. Kerridge JH, Saul P, Lowe, McPhee J, Williams D. Death, dying and donation: organ transplantation and diagnosis of death. J Med Ethics. 2002;28:89-94.

6. Siminoff LA, Lawrence RH. Knowing patients' preferences about organ donation: does it make a difference? J Trauma. 2002;53:754-60.

7. Roza BA. Efeitos do processo de doação de órgãos e tecidos em familiares: intencionalidade de uma nova doação [tese]. São Paulo: Escola Paulista de Medicina, Universidade Federal de São Paulo; 2005.

8. Martinez JM, Martín A, López JS. La opinión pública y el trasplante de órganos. Méd Clin. (Barc) 1995;105:401-6.

9. May T, Aluizio MP, Devita MA. Patientes, families, and organ donation: who should decide? Milbank Q. 2000;48:232-336.

10. Beaulieu D. Organ donation: family's right to make an informed choice. J Neurosci Nurs. 1999;31:37-42

11. West R, Burr G. Why families deny consent to organ donation. Aust Crit Care. 2002;15:27-32.
12. Spital A, Erin CA. Concription of cadaveric organs for transplantation: let's at least talk about it. Am J Kidney Dis. 2002;39:609-10.

13. Associação Brasileira de Transplante de órgãos (ABTO). Registro Brasileiro de Transplante (RBT). Ano XII, n 2, janeiro/dezembro 2006.

14. Massarollo MCKB, Kurcgant P. Vivencial dos enfermeiros no programa de transplante de fígado de um hospital público. Rev Latino-am Enfermagem. 2000;8:66-72.

15. Barber K, Falvey S, Hamilton C, Collett D. Potential for organ donation in the united kingdom: audit of intensive care records. BMJ. 2006;332:1124-7.

16. Sheehy E, Conrad SL, Brigham LE, Luskin R, Weber P, Eakin M, at al. Estimating the number of potential organ donors in the United States. N Engl J Méd. 2003,349(7):667-74.

17. Oliveira JC. Estudo bibliométrico das publicações de custos em enfermagem no período de 1966 a 2000. [dissertação]. São Paulo: Escola de Enfermagem da USP, 2001.

18. Garcia VD. Por uma política de transplante no Brasil. São Paulo: Office; 2000.

19. Padrão MB, Lima AAF, Moraes EL. Fatores que influenciam a recusa familiar que influenciam a recusa familiar no processo doação de órgãos e tecidos para transplante. J Bras Transpl. 2004,7(3)137-40.

20. Lima, AAF. Sofrimento e contradição: o significado da morte, do morrer e da humanização para enfermeiros que trabalham no processo de doação de órgãos para transplante [dissertação]. São Paulo: Centro Universitário São Camilo, Curso de Bioética; 2006.

21. Associação Brasileira de Transplante de órgãos (ABTO). Registro Brasileiro de Transplante (RBT). Ano XI, n 2, janeiro/dezembro 2005.

22. Associação Brasileira de Transplantes de Órgãos. Boletim Informativo. Janeiro/ Junho. 2005;1:14-15.

23. Segre M. A questão ética e a saúde humana. São Paulo: Atheneu, $1^{\mathrm{a}}$ ed. 2006 